\title{
Serum brain-type creatine kinase increases in children with osteogenesis imperfecta during neridronate treatment
}

\author{
Patrizia D'Eufemia', Roberto Finocchiaro', Ciro Villani², Anna Zambrano', Valentina Lodato', Marta Palombaro' ${ }^{1}$ Enrico Properzi ${ }^{1}$ \\ and Mauro Celli ${ }^{1}$
}

BACKGROUND: Creatine kinase (Ck) catalyzes the reversible transfer of high-energy phosphate groups between adenosine triphosphate and phosphocreatine. The brain isoform $(\mathrm{Ckbb})$ is greatly induced in mature osteoclasts, playing an important role in bone-resorbing function during osteoclastogenesis. High Ckbb serum level has been found in patients with osteopetrosis and in patients with bisphosphonate (BP)-induced osteopetrosis. BPs are considered the treatment of choice for children with osteogenesis imperfecta (OI), acting as potent inhibitors of bone resorption by suppressing the activity of osteoclasts.

METHODS: We determined total serum $\mathrm{Ck}$ and isoform activity in 18 prepubertal children with type I OI, before and during treatment with the $\mathrm{BP}$ neridronate infusions.

RESULTS: Basal serum Ckbb levels were slightly elevated with respect to controls (mean $\pm \mathrm{SD}=3.0 \pm 2.7$ vs. 2.0 \pm 2.2 ) and progressively increased after neridronate treatment ( $t_{0}$ vs. $t_{4}$ : mean \pm $\mathrm{SD}=3.0 \pm 2.7$ to $10.8 \pm 8.1)$, with significant increment after first, second, and fourth infusions $(P<0.01)$. An inverse correlation was found between serum Ckbb and serum CTx at basal level.

CONCLUSION: Our results support previous observations that increased serum Ckbb reflects failure of osteoclasts or, at least, suppression of osteoclasts. Upon considering that BPs are long acting, this information could be useful to prevent the risk of overtreatment after long-term BP exposure in pediatric patients with Ol.

C reatine kinase (Ck) catalyzes the reversible transfer of highenergy phosphate groups between adenosine triphosphate (ATP) and phosphocreatine, thereby playing a storage and distribution role in cellular energetics (1). The mitochondrial isoform generates creatine phosphate, which is shuttled to cytosolic isoforms localized to specific subcellular regions to provide ATP at sites where high and fluctuating energies are required (2). There are two mammalian $\mathrm{Ck}$ cytosolic isoforms, the muscle-type $(\mathrm{Ckm})$ and the brain-type $(\mathrm{Ck} b)$, that form homodimers or heterodimers as $\mathrm{Ck} m m, \mathrm{Ck} m b$, and $\mathrm{Ck} b b$. $\mathrm{Ckmm}$ is present in skeletal muscle, and Ckmm, Ckmb, and $\mathrm{Ck} b b$ are present in the heart (1). $\mathrm{Ck} b b$ is present in a range of tissues, including brain, retina, uterus, testes, and osteoclasts, in which it executes the function of energy maintenance and regulation (3).

Mature osteoclasts exhibit high citric acid cycle activity and active mitochondrial respiration in order to generate high levels of ATP, which are used to maintain ATPase activity and ultimately lead to bone resorption (4). Recent studies suggest that $\mathrm{Ck} b b$ is a controlling enzyme in the compartmentalization of ATP availability and that it is greatly induced in mature osteoclasts, playing an important role in bone-resorbing function during osteoclastogenesis (5-7). Studies using $\mathrm{Ck} b b$ null mice have demonstrated that $\mathrm{Ck} b b$ deficiency does not affect basal bone turnover; however, it protects mice from bone loss stimulated by ovariectomy or systemic lipopolysaccharide challenge (6). Furthermore, Ckbb deficiency does not affect osteoclast differentiation but reduces osteoclast activity in vitro, thereby altering bone remodeling (6).

In humans, elevation of serum levels of $\mathrm{Ck} b b$ has been found in some types of osteopetrosis (OPT) in which osteoclasts fail to resorb bone (8-10). Moreover, a study examining patients with the most common clinical forms of OPT and patients with other sclerosing bone disorders found that the presence of $\mathrm{Ck} b b$ in serum seemed to distinguish true OPT (11). Ckbb serum also increased in patients in which drug-induced OPT was a consequence of administration of bisphosphonates (BPs) (12).

Osteogenesis imperfecta (OI) is a genetic disorder with increased bone fragility of varying severity (13). There are four main types: type I is the mildest form without major bone deformities (13). OI is a disease of the osteoblast, producing an abnormal matrix that does not respond to mechanical loads. In compensation, the osteoblast population increases and osteoclast activity is raised, leading to a high bone turnover rate (14). BPs are considered the treatment of choice in pediatric population. Despite clinical evidence of their efficacy, some aspects of the complex mechanism of action remain to be elucidated (15). It is well known that this category of synthetic analogues of inorganic pyrophosphate potently inhibit bone resorption by suppressing the activity of osteoclasts and shortening their life span (16). In this view, it is likely hypothesized that treatment with BPs could promote an alteration of Ckbb activity. 
On the basis of these biochemical and clinical studies, we determined serum $\mathrm{Ck} b b$ activity in children with OI type I, before and during therapy with neridronate, a BP registered in Italy for the treatment of OI (17).

\section{RESULTS}

Results of serum total Ck and isoforms in OI patients and controls are reported in Table 1 . Basal serum Ckbb was slightly but not significantly elevated with respect to controls and was significantly elevated after the first, second, and fourth infusions. At the end of the treatment, serum Ckbb in patients with OI reached values that triplicate basal levels. The results are reported in Figure 1.

Isotypes $\mathrm{Ckmm}$ and $\mathrm{Ckmb}$ did not show any significant change at baseline and during neridronate therapy except for basal $\mathrm{Ckmb}$ level that was higher compared with that in controls.

We did not find any significant alterations of basal $\left(t_{0}\right)$ total serum Ck level compared with that in controls. Three months after first infusion $\left(t_{1}\right)$, total serum $\mathrm{C} k$ showed a significant increase that did not occur after the following infusions.

Demographic, biochemical, and densitometric characteristics are reported in Table 2. As shown, bone mineral density (BMD) $z$-score showed a significant increase after four neridronate infusions $(P<0.01)$, whereas CTx decreased significantly $(P<0.01)$.

Lumbar spine radiograms evidenced no sign of osteosclerosis after treatment. An inverse significant correlation was found between serum Ckbb and CTx at basal level, suggesting that these two parameters are physiologically correlated in patients with OI type I $(r=-0.65 ; P<0.01$; Figure 2$)$.

\section{DISCUSSION}

The main result of this study is that serum $\mathrm{Ck} b b$ activity progressively increases during neridronate therapy in children with OI type I.

The function of $\mathrm{Ck} b b$ in regulating bone resorption activity in mice has been reported by Chang et al. (6), which uncovered a new link between Ckbb activity and osteoclastic bone resorption. In this study, a proteomics approach has been used to identify new proteins involved in the control of osteoclast maturation and function (6). The results showed that isoform Ckbb, whose expression is considerably increased during osteoclastogenesis, regulates activity of mature osteoclasts, including the formation and maintenance of the actin sealing ring and the activities of proton pump (6).

Further molecular studies demonstrated that $\mathrm{Ck} b b$ was the predominant isoform in osteoclasts and that receptor activator of nuclear factor $\mathrm{\kappa B}$ ligand (RANKL) upregulated $\mathrm{Ck} b b$ mRNA expression during osteoclastogenesis (7).

Previous clinical studies reported elevated serum level of $\mathrm{Ck} b b$ in patients with OPT (even clinically mild types) in which osteoclastic dysfunction is the cardinal pathogenetic factor (8-11). In addition to genetic OPT, elevated serum $\mathrm{Ck} b b$ has been reported in acquired OPT in a 12-y-old boy with idiopathic bone pain and treated with very high doses of $\mathrm{BP}$ (pamidronate) over a period of 2 y (12). Evidence of OPT was present $18 \mathrm{mo}$ after his last dose of pamidronate, together with increased serum $\mathrm{Ck} b b$. This patient had a normal serum $\mathrm{Ck} b b$ when he was reevaluated 6 y later, showing that his osteoclasts resumed functioning (18). These findings suggest that elevated serum $\mathrm{Ck} b b$ reflects a fundamental disturbance in osteoclast biology, so that the authors proposed it as a potential marker of osteoclast failure although the mechanisms involved remain unclear $(10,11)$.

It is well known that nitrogen-containing BPs, such as neridronate, potently inhibit skeletal resorption by suppressing the activity of osteoclasts and shortening their life span (16). There is considerable controversy whether BPs can cause oversuppression of bone remodeling, and as a consequence, optimum doses and duration therapy remain unclear $(19,20)$. In fact, in spite of therapeutic efficacy in increasing BMD in skeletal disorders, accumulating reports cautioned that prolonged administration of commonly used doses of BPs might

Table 1. Demographic and clinical characteristics of patients with osteogenesis imperfecta type I given neridronate treatment

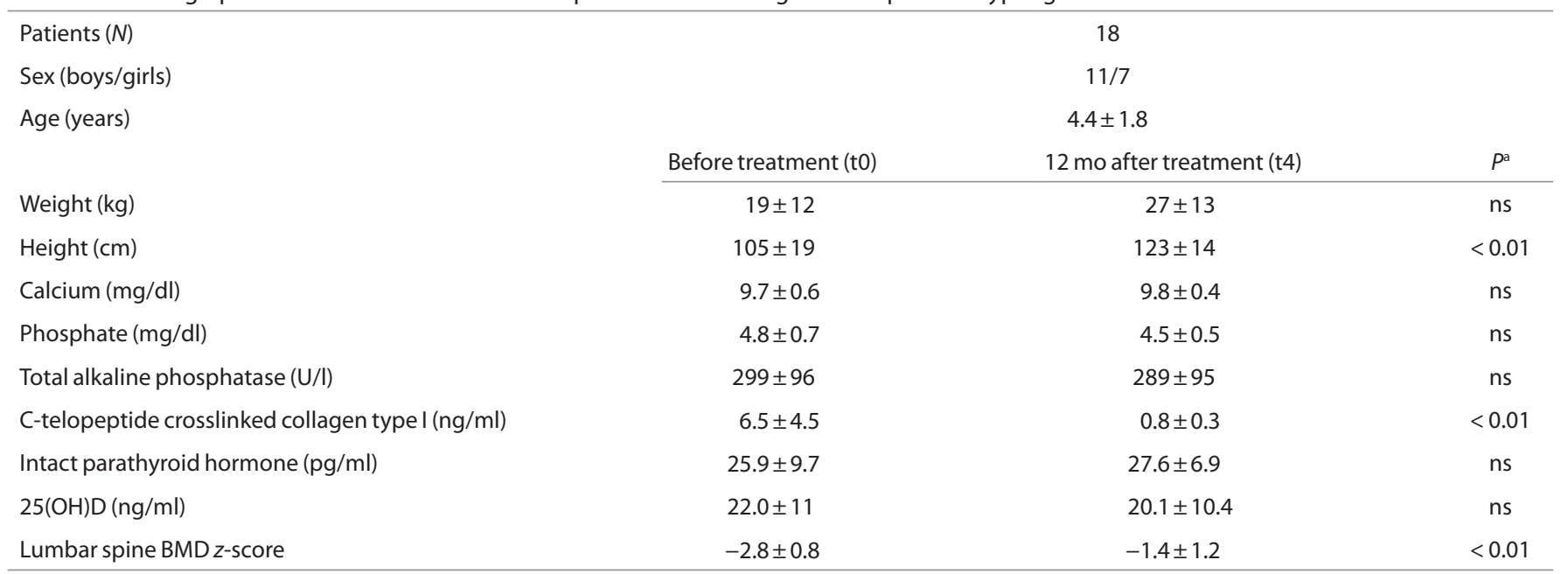

Values are given as mean \pm SD

BMD, bone mineral density; ns, not significant.

apaired $t$-test. 


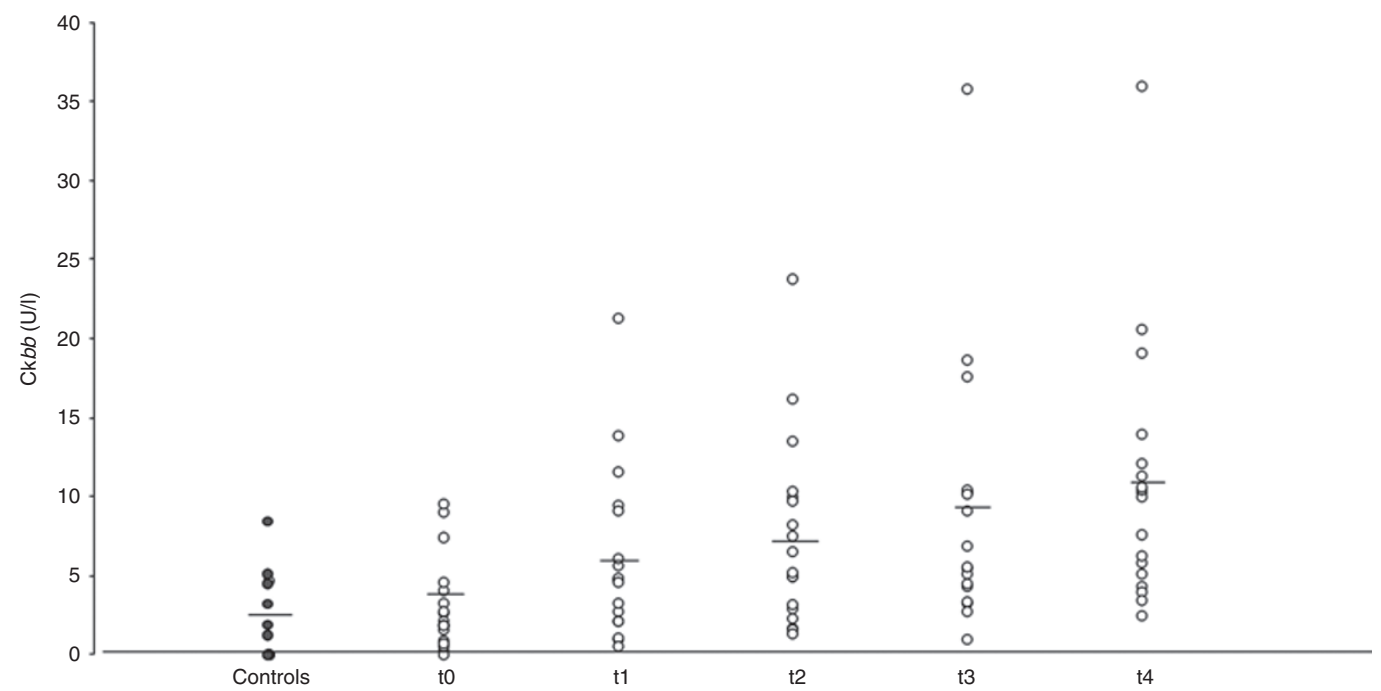

Figure 1. Serum Ckbb values in controls $(\bullet)$ and patients with osteogenesis imperfecta type I $(O)$ at baseline $\left(t_{0}\right)$ and during neridronate treatment $\left(t_{1}-t_{4}\right)$.

Table 2. Serum total $\mathrm{Ck}$ and isoforms levels in osteogenesis imperfecta type I patients given neridronate treatment $\left(t_{0}-t_{4}\right)$

\begin{tabular}{|c|c|c|c|c|c|c|c|c|c|c|c|}
\hline \multirow[b]{2}{*}{ Ck isoforms } & \multirow[b]{2}{*}{$\begin{array}{l}\text { Control } \\
\text { group }\end{array}$} & \multicolumn{5}{|c|}{ Patients } & \multicolumn{5}{|c|}{$P^{a}$} \\
\hline & & $t_{0}$ & $t_{1}$ & $t_{2}$ & $t_{3}$ & $t_{4}$ & $\begin{array}{l}t_{0} \text { vs. control } \\
\text { group }\end{array}$ & $t_{1}$ vs. $t_{0}$ & $t_{2}$ vs. $t_{1}$ & $t_{3}$ vs. $t_{2}$ & $t_{4}$ vs. $t_{3}$ \\
\hline $\mathrm{Ck} b b(\mathrm{U} / \mathrm{l})$ & $2.0 \pm 2.2$ & $3.0 \pm 2.7$ & $6.1 \pm 5.3$ & $7.5 \pm 5.9$ & $9.2 \pm 8.8$ & $10.8 \pm 8.1$ & \multirow{2}{*}{0.09} & \multirow{2}{*}{$<0.01$} & \multirow{2}{*}{$<0.01$} & \multirow{2}{*}{0.02} & \multirow{2}{*}{$<0.01$} \\
\hline$\%$ & $1.8 \pm 3.1$ & $3.9 \pm 3.3$ & $6.5 \pm 4.4$ & $7.3 \pm 6.6$ & $7.3 \pm 5.8$ & $6.1 \pm 3.6$ & & & & & \\
\hline $\mathrm{Ckmm}(\mathrm{U} / \mathrm{l})$ & $86 \pm 10$ & $82 \pm 33$ & $101 \pm 29$ & $101 \pm 27$ & $109 \pm 41$ & $105 \pm 28$ & \multirow{2}{*}{0.84} & \multirow{2}{*}{0.05} & \multirow{2}{*}{0.97} & \multirow{2}{*}{0.19} & \multirow{2}{*}{0.34} \\
\hline$\%$ & $97.0 \pm 5.5$ & $92.0 \pm 3.8$ & $89.4 \pm 3.2$ & $87.9 \pm 6.1$ & $89.9 \pm 5.3$ & $89.9 \pm 3.4$ & & & & & \\
\hline
\end{tabular}

Values are given as mean \pm SD.

Ck, creatine kinase

apaired $t$-test.

impair bone modeling and remodeling and induce OPT-like changes, with structural changes that evolve and carry out into adult life $(18,19)$.

Our study provides data on basal serum $\mathrm{Ck} b b$ in children with OI type I and early changes of basal serum Ckbb in relation to neridronate treatment. Basal serum $\mathrm{Ck} b b$ showed a slight but not significant elevation with respect to the normal controls. This finding is in accordance with the pathogenesis of OI in which molecular defect affects the osteoblast function linked to a compensatory raise of osteoclast activity. Regarding other isoforms at basal level ( $\mathrm{t} 0$ ), we found a significant increase of the specific cardiac isoform $(\mathrm{Ckmb})$. We are not able to explain this result because our patients were screened for the absence of cardiovascular diseases.

After the first neridronate infusion, we observed a significant increase of serum $\mathrm{Ck} b b$, a result that seems to evidence the effectiveness of this biochemical marker in revealing early osteoclast failure induced by BP. Moreover, serum $\mathrm{Ck} b b$ showed a progressive increase after each infusion that could reflect the cumulative effect of BP in suppressing osteoclast function. Of interest, at basal level $\left(t_{0}\right)$, we found an inverse correlation between serum $\mathrm{Ck} b b$ and $\mathrm{CTx}$, a biochemical parameter considered a reliable indicator of in vivo osteoclast activity representing one of the end products of bone matrix resorption. This result reinforces the hypothesis that the determination of serum $\mathrm{Ck} b b$ could be considered as a new molecular target for osteoclast failure $(10,11)$. Nevertheless, after the first infusion, no significant correlation was still present between these two parameters. The lack of this correlation might be explained on the basis of different responses of $\mathrm{Ck} b b$ and CTx to the treatment with BP. In fact, whereas $\mathrm{Ck} b b$ showed a progressive increase, CTx rapidly decreased just after 3 mo of treatment, maintaining successively low levels. This trend is in agreement with the results obtained in recent studies performed on biochemical markers in patients with OI treated with BPs $(21,22)$. Taken together, these results could suggest that the osteoclasts are fully suppressed once the first infusion has been administered.

Our results support previous observations that increased serum $\mathrm{Ck} b b$ reflects failure of osteoclasts or, at least, suppression of osteoclasts. More detailed investigation is going to be necessary to understand the significance and utility of a rising or increased $\mathrm{Ck} b b$ level in the management of OI with BPs or 


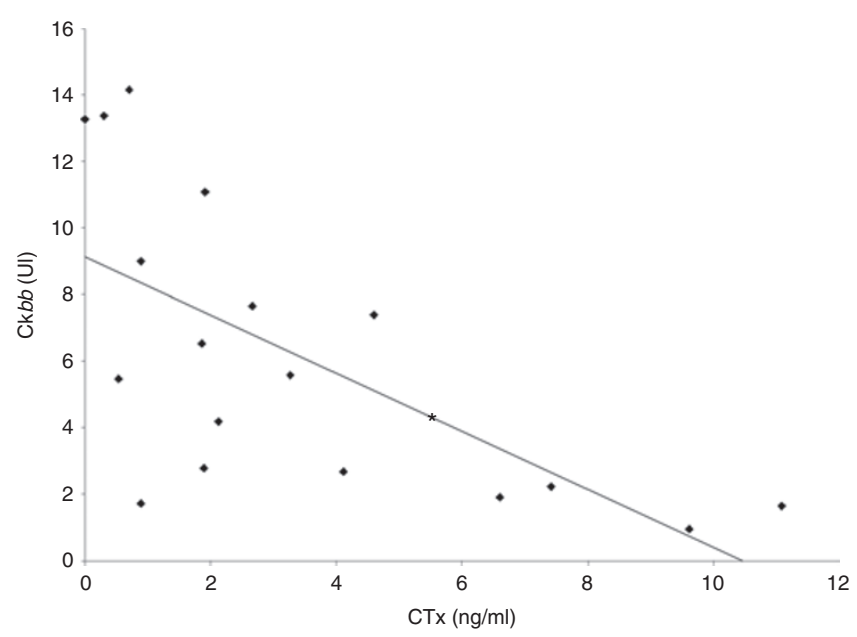

Figure 2. $C k b b$ activity and CTx levels in serum of 18 children with osteogenesis imperfecta type I before neridronate treatment (t0) $(\bullet)$. ${ }^{*} P<0.001$ (linear regression analysis); regression line is shown $(r=-0.65)$.

even in the management of other metabolic bone diseases. In this view, further studies on bone histomorphometry and histology that look for evidence of OPT are needed. Upon considering that BPs are long-acting drugs, this information could be useful to prevent the risk of overtreatment in pediatric OI patients after long-term exposure to BPs.

\section{METHODS}

\section{Study Subjects}

Written informed consent was obtained from the parents of the patients before inclusion of patients in the study. This study protocol was approved by the ethics committee of the Department of Pediatrics, "Sapienza," University of Rome. Eighteen prepubertal children with the mildest form of OI (type I), i.e., 11 boys and 7 girls aged $2-8.7$ y (mean \pm SD: $4.4 \pm 1.8$ ) treated as outpatients in the Department of Pediatrics, "Sapienza," University of Rome were enrolled in the study (Table 2). Patients were diagnosed based on clinical and radiological features reported in the classification by Sillence et al. (23). In all patients, the clinical diagnosis was confirmed by molecular DNA analysis that evidenced mutations of the COL1A1 gene.

Exclusion criteria were (i) history of treatment with BPs or drugs affecting the bone metabolism and (ii) gastrointestinal, cardiovascular, or neuromuscular diseases inducing $\mathrm{Ck}$ alteration. Cardiovascular diseases were screened by ECG and echocardiography. Inclusion criteria were (i) prepubertal age and (ii) vertebral compression fractures, as selection criteria for starting treatment.

All patients had their dietary calcium intake regularly evaluated and maintained through the diet or supplementation of 600-800 mg according to their age. Vitamin D3 supplements were given when serum $25(\mathrm{OH})$ vitamin D levels were below the lower limit of normal range established in our laboratory (32 $\mathrm{ng} / \mathrm{ml})$.

The treatment was started with a single neridronate infusion once in 3 mo for a period of 12 mo (t0-t4). Each infusion consisted of $2 \mathrm{mg}$ neridronate/ $\mathrm{kg}$ body weight, diluted in
$100 \mathrm{ml}$ isotonic saline. At baseline $\left(t_{0}\right)$ and just before each infusion $\left(t_{1}-t_{4}\right)$, according to our protocol treatment for OI, auxological data were registered, and serum biochemical markers of bone metabolism (calcium, phosphate, intact parathyroid hormone (iPTH), 25( OH) vitamin $\mathrm{D}$, total alkaline phosphatase (ALP), C-terminal telopeptide (CTx)), were assayed. In the same samples, we determined serum total $\mathrm{Ck}$ and isoforms. Because, to our knowledge, there are no reference values for serum $\mathrm{Ck}$ isoforms in children, we measured serum $\mathrm{Ck}$ isoforms in a control group consisting of 20 healthy prepubertal children matched for sex and age (12 boys and 8 girls; age: $1-9$ y; mean \pm SD: $4.3 \pm 1.69)$. We also analyzed BMD $z$-score at baseline $\left(t_{0}\right)$ and after 6 and 12 mo of therapy ( $t_{2}$ and $t_{4}$, respectively) in order to investigate a possible correlation with serum isoform changes during neridronate treatment. Lumbar spine radiograms were obtained before and after 12 mo of therapy. We did not perform bone biopsies to look for evidence of osteoclast failure or OPT because this was not a prospective part of our study. For the same reason, we did not perform radiographic studies looking for significant alterations of bone modeling in the wrists or knees.

\section{Serum Biochemical Studies}

Fasting blood was taken in the control group and in patients with OI. Total Ck activity in serum was measured using kinetic UV method (Instrumentation Laboratory SpA, Milano, Italy) optimized according to the Federation of Clinical Chemistry (24). Isoforms of Ck ( $m m, m b$, and $b b)$ were separated by electrophoretics on agarose gels and then incubated for $1 \mathrm{~h}$ at 37 ${ }^{\circ} \mathrm{C}$ before performing spectrophotometric measurement as described previously (25), and isotype activity was expressed as international units per liter (IU/l) (Helena Biosciences Europe, Sunderland, UK). Serum calcium, phosphate, ALP, iPTH, and $\mathrm{CTx}$, and 25(OH) D levels were measured by automatic analyzer and chemiluminescence (Roche diagnostic SpA, Monza, Italy), respectively.

\section{Radiological Studies}

BMD $z$-score was measured at lumbar spine (L1-L4) by dualenergy x-ray absorptiometry using Hologic QDR 4500A system with reference values provided by the manufacturer (Hologic, Bedford, MA) (26).

\section{Statistics}

Results were expressed as mean \pm SD. We assessed statistical significance of the within-subject changes of demographic, densitometric, and biochemical parameters during neridronate therapy by the two-tailed Student's $t$-test. Given that the data were approximately log-normally distributed, they were log-transformed before statistical analysis. Because the variance of the values of serum $\mathrm{Ck} b b$ in the subjects with OI was greater than that of controls, we have applied the MannWhitney nonparametric test to compare them.

Correlations between serum $\mathrm{Ck} b b$ and biochemical markers of bone metabolism were performed by linear regression analysis. Significance was established for $P<0.01$. 


\section{ACKNOWLEDGMENTS}

The authors thank Renzo Guglielmi (Laboratory of the Department of Neurology and Psychiatry, "Sapienza," University of Rome) who provided expert technical assistance and reviewed the Methods section.

\section{STATEMENT OF FINANCIAL SUPPORT}

No financial assistance was received to support this study.

Disclosure: The authors state that they have no conflicts of interest.

\section{REFERENCES}

1. Wallimann T, Tokarska-Schlattner M, Schlattner U. The creatine kinase system and pleiotropic effects of creatine. Amino Acids 2011;40:1271-96.

2. Wallimann T, Dolder M, Schlattner U, et al. Some new aspects of creatine kinase $(\mathrm{CK})$ : compartmentation, structure, function and regulation for cellular and mitochondrial bioenergetics and physiology. Biofactors 1998;8:229-34.

3. Wallimann T, Wyss M, Brdiczka D, Nicolay K, Eppenberger HM. Intracellular compartmentation, structure and function of creatine kinase isoenzymes in tissues with high and fluctuating energy demands: the 'phosphocreatine circuit' for cellular energy homeostasis. Biochem J 1992;281 (Pt 1):21-40.

4. Francis MJ, Lees RL, Trujillo E, Martín-Vasallo P, Heersche JN, Mobasheri A. ATPase pumps in osteoclasts and osteoblasts. Int J Biochem Cell Biol 2002;34:459-76.

5. Kuiper JW, van Horssen R, Oerlemans F, et al. Local ATP generation by brain-type creatine kinase (CK-B) facilitates cell motility. PLoS One 2009;4:e5030.

6. Chang EJ, Ha J, Oerlemans F, et al. Brain-type creatine kinase has a crucial role in osteoclast-mediated bone resorption. Nat Med 2008;14:966-72.

7. Chen J, Sun Y, Mao X, Liu Q, Wu H, Chen Y. Rankl up-regulates brain-type creatine kinase via poly (ADP-ribose) polymerase-1 during osteoclastogenesis. J Biol Chem 2010;285:3615-21.

8. Yoneyama T, Fowler HL, Pendleton JW, et al. Elevated levels of creatine kinase BB isoenzyme in three patients with adult osteopetrosis. N Engl J Med 1989;320:1284-5.

9. Gram J, Antonsen S, Hørder M, Bollerslev J. Elevated serum levels of creatine kinase $\mathrm{BB}$ in autosomal dominant osteopetrosis type II. Calcif Tissue Int 1991;48:438-9.

10. Yoneyama T, Fowler HL, Pendleton JW, et al. Elevated serum levels of creatine kinase $\mathrm{BB}$ in autosomal dominant osteopetrosis type II-a family study. Clin Genet 1992;42:39-42.
11. Whyte MP, Chines A, Silva DP Jr, Landt Y, Ladenson JH. Creatine kinase brain isoenzyme (BB-CK) presence in serum distinguishes osteopetroses among the sclerosing bone disorders. J Bone Miner Res 1996;11:1438-43.

12. Whyte MP, Wenkert D, Clements KL, McAlister WH, Mumm S. Bisphosphonate-induced osteopetrosis. N Engl J Med 2003;349:457-63.

13. Rauch F, Glorieux FH. Osteogenesis imperfecta. Lancet 2004;363:1377-85.

14. Glorieux FH. Osteogenesis imperfecta. A disease of the osteoblast. Lancet 2001;358:S45.

15. Maruotti N, Corrado A, Neve A, Cantatore FP. Bisphosphonates: effects on osteoblast. Eur J Clin Pharmacol 2012;68:1013-8.

16. Russell RG, Watts NB, Ebetino FH, Rogers MJ. Mechanisms of action of bisphosphonates: similarities and differences and their potential influence on clinical efficacy. Osteoporos Int 2008;19:733-59.

17. Adami S, Gatti D, Colapietro F, et al. Intravenous neridronate in adults with osteogenesis imperfecta. J Bone Miner Res 2003;18:126-30.

18. Whyte MP, McAlister WH, Novack DV, Clements KL, Schoenecker PL, Wenkert D. Bisphosphonate-induced osteopetrosis: novel bone modeling defects, metaphyseal osteopenia, and osteosclerosis fractures after drug exposure ceases. J Bone Miner Res 2008;23:1698-707.

19. Adami S, Zamberlan N. Adverse effects of bisphosphonates. A comparative review. Drug Saf 1996;14:158-70.

20. Rauch F, Glorieux FH. Bisphosphonate treatment in osteogenesis imperfecta: which drug, for whom, for how long? Ann Med 2005;37:295-302.

21. Aström E, Magnusson P, Eksborg S, Söderhäll S. Biochemical bone markers in the assessment and pamidronate treatment of children and adolescents with osteogenesis imperfecta. Acta Paediatr 2010;99:1834-40.

22. Li M, Xia WB, Xing XP, et al. Benefit of infusions with ibandronate treatment in children with osteogenesis imperfecta. Chin Med J (Engl) 2011;124:3049-53.

23. Sillence DO, Senn A, Danks DM. Genetic heterogeneity in osteogenesis imperfecta. J Med Genet 1979;16:101-16.

24. IFCC Primary reference procedures for the measurements of catalytic activity concentration of enzymes at $37^{\circ} \mathrm{C}$. Part 2: reference procedure for the measurements of catalytic concentration of creatine kinase. Clin Chem Lab Med 2002;40:635-42.

25. Trainer TD, Gruenig D. A rapid method for the analysis of creatine phosphokinase isoenzymes. Clin Chim Acta 1968;21:151-4.

26. Zemel BS, Kalkwarf HJ, Gilsanz V, et al. Revised reference curves for bone mineral content and areal bone mineral density according to age and sex for black and non-black children: results of the bone mineral density in childhood study. J Clin Endocrinol Metab 2011;96:3160-9. 